ORIGINAL ARTICLE

\title{
The Effect of Nursing Protocol in Insertion and Care of Vascular Access Devices in Oncology Pediatric Patients
}

\author{
HANAN MARZOUK ${ }^{1}$, IMAN IBRAHIM ABDEL-MONEIM², HYAM REFAAT TANTAWI ${ }^{3}$ \\ M.SC ${ }^{(1)}$, Professor of Pediatric Nursing ${ }^{(2,3)}$ \\ Faculty of Nursing, Ain Shams University
}

\begin{abstract}
Arrangement of a focal line consistently conveys likely dangers for the patient, with significant contrasts in the sort and seriousness of entanglement, contingent upon which vein, which strategy and which gadget have been picked.

Aim: The current study aimed to study the effect of nursing protocol in insertion and care of Vascular Access Devices (VAD) in oncology pediatric patients

Design: A quasi experimental design was used.

Setting: This study was conducted at the oncology department Unit, affiliated to Ain Shams University Hospital and police hospital at El-Agoza.

Sample: A purposive example of (50) nurses were working at Ain Shams and (20) at EIAgoza. I. Predesigned Questionnaire Format, Assessment tool for the studied children, Questionnaire tool for nurses to assess nurse's knowledge, Observational checklists used to assess the nurses' practices regarding nursing protocols for care and insertion of VAD

Results: there was positive correlation between knowledge of the studied nurses and their total practices and regarding vascular access devices (Port-A-Cath, Hickman, and CVC) throughout intervention with statically significant differences $(p<0.05)$.

Conclusion: Implementation of the nursing protocol has statistically significant positive effect in insertion and care of VAD on oncology pediatric patients

Recommendation: the nursing protocol should be applied for all nurses who deal with pediatric devices.

Keywords: Nursing Protocol, Vascular Access Devices, Oncology, Pediatric Patients
\end{abstract}

\section{INTRODUCTION}

In late decades, the expanding utilization of Vascular Access Devices (VADs), either Long Term (LTVA) or Medium Term (MTVA), has given a critical improvement in the treatment of youngsters influenced by hematologic illnesses. As indicated by the as of now acknowledged phrasing, LTVA is characterized as a VAD with specialized highlights which increment its steadiness in time, with a normal length in the scope of months and years: this class incorporates burrowed bound catheters and absolutely implantable venous ports. MTVA are VADs fitting for a drawn out yet not boundless time (weeks or months) and for a broken use: which incorporate burrowed nonhandcuffed focal catheters, Peripherally Inserted Central Catheters (PICCs) and midline catheters (Gallieni et al., 2018).

A dependable and durable focal VAD grants a more secure and simpler organization of chemotherapy, strong medications, hyper hydration and hypertonic arrangements, for example, all out parenteral nourishment, than by means of the fringe vein. Position of a focal VAD can be performed by specialists, anesthesiologists, intensivists, interventional radiologists, oncologists or even medical attendants, contingent upon the sort of gadget and the decision of venous access. (Randolph et al., 2013)

LTVA and MTVA can be put with various methods (venous chop down, "daze" percutaneous venipuncture guided by anatomical tourist spots, ultrasound-guided percutaneous cut and cannulation of the vein). Arrangement of a focal line consistently conveys likely dangers for the patient, with significant contrasts in the sort and seriousness of entanglement, contingent upon which vein, which strategy and which gadget have been picked (Braveman et al., 2012).

An oncology medical attendants engaged with thinking about kids with vascular access gadgets ought to be prepared, and surveyed as skillful, in utilizing and reliably clinging to these rules and convention to limit the disease hazard related with overseeing VAD to give uniform direction over the dependent on best proof, to furnish medicinal services staff with the information, to oversee VAD securely and instruction of kids and, their Caregivers (Barnes et al., 2014).

Convention is expected to give proof based suggestions to forestalling diseases identified with VAD. Significant regions of accentuation incorporate instructing and preparing medicinal services faculty who embed and keep up catheters and utilizing maximal clean boundary precautionary measures during VAD inclusion and care (Strootman, V. 2014).

Significance of the problem: Serious conditions of the complication related insertion of the devices of oncology pediatric patients were recorded so that it was the time to investigate and improve the performance of nursing staff regarding this invasive procedures and prevent its complication specially that related infection and to focus on the factors that leading to these complications. Many cases at the oncology department suffering from complication from the VAD by hospital records the researcher found that every week there was at least two cases suffering from complication from VAD care.

Aim of the Work: The current study aimed to study the effect of nursing protocol in insertion and care of VAD in oncology pediatric patients 
Research hypothesis: The nursing protocol will effect positively in insertion and care of VAD on oncology pediatric patients.

\section{SUBJECTS AND METHODS}

This investigation was planned to evaluate the impact of nursing convention in inclusion and care of VAD in oncology pediatric patients through the accompanying goals:

- Assessing medical attendants' information and work on with respect to nursing convention in inclusion and care of VAD in oncology pediatric patients.

- $\quad$ Designing, actualizing and assessing the nursing convention in addition and care of VAD in oncology pediatric patients

Research hypothesis: The subject and methods of the current study discussed under the following four (4) designs:

Research Design

Operational Design

Administrative Design

Statistical Design

Sample

A purposive example of (50) nursing staff, who were working at the recently referenced setting and give care to children affirmed determination with oncology and have VAD or will embed VAD.

Predesigned Questionnaire Format: The specialist was created it in the wake of looking into the important literary works. It was written in basic Arabic language. It was planned by the analyst subsequent to checking on the current accessible writing and was written in straightforward Arabic language to survey the accompanying parts:

- Assessment tool for the studied children : It was concerned with:

- Demographic qualities of the considered kids as sex, age, level of instruction and positioning

- Characteristics of youngsters experiencing oncology as past emergency clinic affirmations because of oncology, reasons for confirmation and kind of chemotherapy the board with VAD.

- Questionnaire instrument for medical caretakers to survey attendant's information: This apparatus was created by the scientist in the light of applicable references and made out of the accompanying parts:

- $\quad$ First part was worried about attributes of the medical caretakers, for example, age, level of instruction, long stretches of understanding and participation of past preparing about nursing conventions for the board of kids with VAD.

- Second part: This part was utilized to survey information in regards to of was worried about the attendants' information about VAD. It Contained 50 various decision inquiries regarding:

Scoring system: As indicated by the reactions got from the nurse's, a scoring framework was followed to evaluate the attendant's information each question scored one (1) for right answer and each erroneous answer, scored zero (0). The score of inquiries were summarized and the all out isolated by number of the things, giving a mean score. These scores were changed over into a percent score was named the accompanying:

$<75$ unsatisfactory knowledge.

$\geq 75$ satisfactory knowledge

II. Observational agendas: It was embraced from (Nursing Protocol 57357) (2015) and was utilized to survey the attendants' works on in regards to nursing conventions for care and inclusion of VAD:

- $\quad$ Preparation for the vascular access gadgets addition.

- Care during addition the vascular access gadgets.

- Care after the vascular access gadgets inclusion.

- Port-a-cath needle inclusion.

- Daily care and support of the vascular access gadgets.

- Flushing with heparin/saline of the vascular access gadgets.

- Obtaining blood examples from the vascular access gadgets.

- Administration of liquid implantation through the vascular access gadgets.

- Administration of chemotherapy through the vascular access gadgets.

- Antibiotic lock for the vascular access gadgets.

- Vascular get to gadgets expulsion.

Scoring system: The correct advance was scored one, and that wrong was scored zero. The score of steps were summarized and the all out separated by number of the means, giving a mean score. These scores were changed over into a percent score was named the accompanying: $<80$ incompetent practices

$\geq 80$ competent practices

\section{II- Operational Design:}

- Preparatory phase:

- This stage included checking on of writing identified with of nursing convention in addition and care of VAD in oncology pediatric patients. This served to build up the investigation apparatuses for information assortment. During this stage, the analyst additionally visited the chose spots to be familiar with the staff and the investigation settings. Improvement of the devices was under directors' direction and specialists' suppositions considered.

Face and content validity: Content legitimacy was learned by a gathering (5) of the specialists in field of pediatric nursing to test its substance legitimacy and relevance, Reliability was wear utilized test-retest $(0.82)$.

Pilot Study: Pilot study did on 10\% (5 attendants) of medical caretakers were working at the recently referenced setting to test the relevance of the developed apparatuses and the lucidity of the included inquiries related of nursing convention in addition and care of VAD in oncology pediatric patients. The pilot has likewise served to evaluate the time required for each subject to fill in the inquiries. As indicated by the consequences of the pilot, a few amendments, for example, rethinking and included inquiries proceeded varying. The pilot members were excluded from the primary examination test.

Fieldwork: The real field work of this investigation was completed more than half year's time frame expressed from the earliest starting point of January 2019 till the finish of June 2019. The specialist was accessible in the investigation setting three days out of every week in the first part of the day move. Each medical attendant was 
talked with separately to assemble the essential information of the investigation. As respects the attendants' practices, it was evaluated by the analyst all through their every day care in the investigation setting.

\section{The present study was conducted in four phases:}

- Preparatory stage: The device of information assortment advancement An audit of the past and current related writing covering different parts of cell phone sound use, utilizing accessible books, periodicals, articles and magazines. The targets were to get to know the examination issue to build up the investigation apparatuses.

- Assessment stage: By utilizing pre-testing poll to survey the current medical caretaker's information and practices about nursing care of VAD in oncology pediatric patients.

- Planning and execution stage: By building up the nursing convention of inclusion and care of VAD in oncology pediatric patients and actualizing it:

The wellbeing instructive general target was to improve the nursing medical attendants' information with respect to nursing convention in addition and care of VAD in oncology pediatric patients.

Based on the consequence of the pre-test survey, the scientists used various meetings (hypothesis and practice) went from 7-10 meetings and every meeting required from 1-2 hours, and meeting with medical caretakers was two days out of every week.

- Nursing medical attendants were separated into 5 gatherings, and each gathering comprised of 10 medical caretakers.

The nursing convention in addition and care of VAD in oncology pediatric patients content assisted each medical attendant to be capable with defining: the VAD, clarify the signs for inclusion of AVD, examining nursing job previously, during and after addition, distinguishing the term of addition, explaining the intricacies of catheters and contraindications for inclusion.

The nursing convention in inclusion and care of VAD in oncology pediatric patients was customized to suit nursing medical caretakers' instructive needs. The analyst built up an Arabic delineated booklet which coordinated the medical caretakers about cell phone solid use.

Evaluation stage: This stage meant to assess the impact of nursing convention in inclusion and care of VAD in oncology pediatric patients. One post-test administrated to the examination subjects was quickly performed after culmination of the nursing convention in inclusion and care of VAD in oncology pediatric patients to assess the maintenance of information and works on with respect to mind of VAD in oncology pediatric patients.

After a half year of executing the nursing convention in addition and care of VAD in oncology pediatric patients a subsequent evaluation incorporated the perception agendas referenced already and utilized in the preappraisal, which was accomplished for all medical caretakers. The specialists watched the attendant after the preparation program has been done to know how the advantage of the convention and their use of nursing convention in inclusion and care of VAD in oncology pediatric patients and what are the methods of obstructions to absence of execution.

\section{Administrative Design}

- Endorsement got through on gave letter from the Dean of Faculty of Nursing, Ain Shams University to overseers of the recently referenced settings. The analyst at that point met the emergency clinic chief and clarified the reason and the techniques for the information assortment.

\section{Moral thought}

- The examination endorsement got from the staff moral board before beginning the investigation. Verbal endorsement acquired from the medical caretakers before consideration in the investigation; a reasonable and straightforward clarification offered by their degree of understanding, physical and mental preparation. They made sure about that all the assembled information was classified and utilized for research reason as it were. The medical attendants educated that they are permitted to decide to take an interest or not in the investigation and they reserve the privilege to pull back from the examination whenever.

\section{Statistical Design:}

- Information gathered from the considered example was reconsidered, coded and entered utilizing. PC. Automated information section and measurable investigation were satisfied utilizing the factual bundle for sociologies (SPSS) variant 22.Data were introduced utilizing graphic measurements as frequencies, rates. Chisquare test(X2) utilized for examinations between subjective factors and connection cofficiency was utilized to test relationship between's factors. Factual huge was considered at $\mathrm{p}$-esteem $<0.05$.

\section{Part I: Demographic Characteristics of the Studied Sample}

\section{(A): Characteristics of the Studied Nurses}

Table (1): Distribution of the studied nurses according to their characteristics $(\mathrm{no}=50)$

\begin{tabular}{|l|l|l|}
\hline Items & No & $\%$ \\
\hline Gender & \multicolumn{1}{l|}{} \\
\hline Male & 12 & 24 \\
\hline Female & 38 & 86 \\
\hline Age in years & & \\
\hline$<20$ & 2 & 4 \\
\hline $20<25$ & 13 & 26 \\
\hline $25<30$ & 16 & 32 \\
\hline $30<35$ & 9 & 18 \\
\hline $35<40$ & 7 & 14 \\
\hline$\geq 40$ & 3 & 6 \\
\hline Mean \pm SD & $27.4 \pm 2.8$ & \\
\hline Qualification & \multicolumn{1}{|l|}{} \\
\hline Diploma & 16 & 32 \\
\hline Diploma \& specialty & 8 & 16 \\
\hline Technical institute & 9 & 18 \\
\hline BSN & 13 & 26 \\
\hline Neonatal nursing scholarship & 4 & 8 \\
\hline Years of experience & \multicolumn{1}{|l|}{} \\
\hline$<5$ & 6 & 12 \\
\hline $5<10$ & 23 & 46 \\
\hline $10<15$ & 12 & 24 \\
\hline$\geq 15$ & 9 & 8 \\
\hline
\end{tabular}

Table (1) shows that, the majority of the studied nurses were females (86\%), 32.0\% of them were in the age group of $25<30$ years and $32 \%$ were diploma nurses. Regarding 
years of experience, almost half (46\%) of them were had years of experience between $5<10$.

Figure (1): Distribution of the studied nurses according to their previous training $(\mathrm{no}=50)$

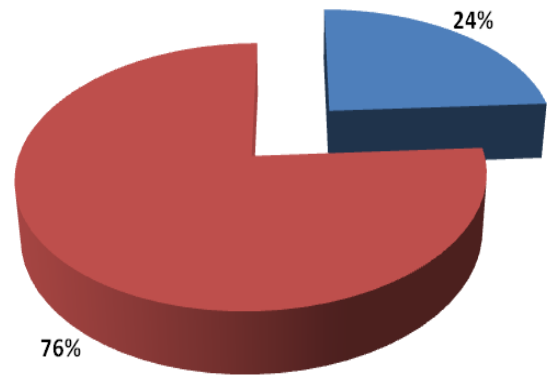

- Yes No

Figure (1) shows that, three quarters $(76 \%)$ of the studied nurses didn't attend a training program regarding vascular access devices.

\section{(B): Characteristics of the Studied Children}

Table (2): Distribution of the socio-demographic characteristics of the studied children $(\mathrm{no}=50)$

\begin{tabular}{|c|c|c|}
\hline Item & No & $\%$ \\
\hline $\begin{array}{l}\text { Gender } \\
\text { Male } \\
\text { Female }\end{array}$ & $\begin{array}{l}29 \\
21\end{array}$ & $\begin{array}{l}58 \\
42\end{array}$ \\
\hline $\begin{array}{l}\text { Age \year } \\
<1 \\
1-3 \\
4-6 \\
7-9 \\
10-12 \\
13-15 \\
16-18 \\
\text { Mean } \pm \text { SD }\end{array}$ & $\begin{array}{l}2 \\
3 \\
9 \\
13 \\
7 \\
6 \\
10 \\
(8.6 \pm 0.6)\end{array}$ & $\begin{array}{l}4 \\
6 \\
18 \\
26 \\
14 \\
12 \\
20\end{array}$ \\
\hline $\begin{array}{l}\text { Level of educational } \\
\text { Preschool } \\
\text { Primary school } \\
\text { Preparatory school } \\
\text { Secondary school }\end{array}$ & $\begin{array}{l}5 \\
27 \\
8 \\
10\end{array}$ & $\begin{array}{l}10 \\
54 \\
16 \\
20\end{array}$ \\
\hline
\end{tabular}

Table (2) : illustrates that, more than half (58\%) of studied children were males, the age of $26 \%$ of them ranged between $7-9$ years and more than half $(54 \%)$ were in primary school.

Figure (2): Distribution of the studied children according to their ranking in their family $(\mathrm{no}=50)$

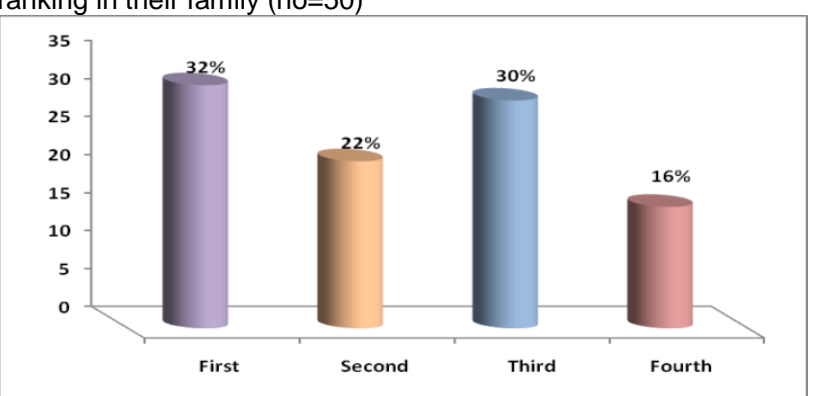

Figure (2) shows that, almost one third (32.0\%) of studied children were ranked as the first child, in their families.
Figure (3): Distribution of the studied nurses regarding their total knowledge about vascular access devices throughout the intervention (no=50)

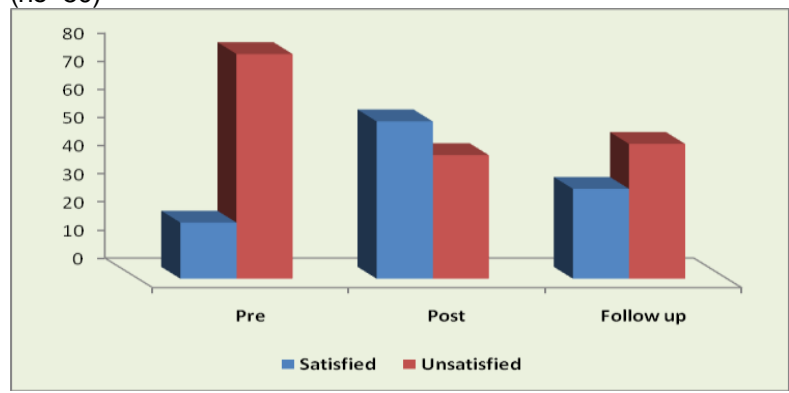

Figure (4): Distribution of the studied nurses according to their practice regarding preparation for the vascular access devices insertion (Port-A-Cath, Hickman, and CVC) throughout intervention $(\mathrm{nO}=50)$

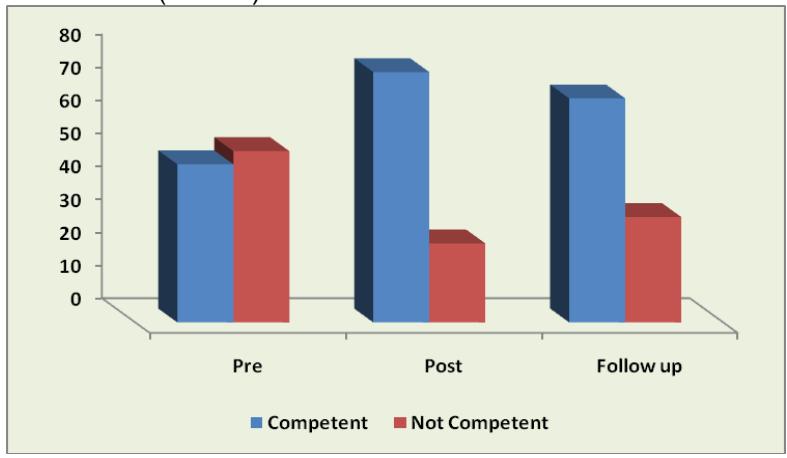

Table (3): Correlation between knowledge of the studied nurses and their total practices and regarding vascular access devices (Port-A-Cath, Hickman, and CVC) throughout intervention (no= 50)

\begin{tabular}{|l|l|l|}
\hline \multirow{2}{*}{ Practices } & \multicolumn{2}{|l|}{ Knowledge } \\
\cline { 2 - 3 } & $\mathrm{r}$ & P-Value \\
\hline pre & 0.43 & 0.8 \\
\hline post & 0.87 & 0.001 \\
\hline Follow up & 0.79 & 0.03 \\
\hline
\end{tabular}

Table (3) illustrates that, there was positive correlation between knowledge of the studied nurses and their total practices and regarding vascular access devices (Port-A-Cath, Hickman, and CVC) throughout intervention with statically significant differences $(p<0.05)$

\section{DISCUSSION}

Before the development of central venous access devices (VADs), children undergoing therapy for cancer faced multiple intravenous (IV) punctures for the administration of therapy and the sampling of blood. Difficulties with establishing and maintaining peripheral venous access complicated the delivery of care both medically and psychosocially, sometimes to the point of therapy being interrupted or stopped. Today the use of VADs has made a tremendous impact on children who are being treated for malignancies, offering many patients a safe, comfortable, and reliable alternative to peripheral venous access (Hickman, et al., 2013).

Broviac catheters were first introduced in 1973 for patients who required long-term hyperalimentation. Hickman and his colleagues in 1976 introduced a larger, more versatile catheter that could be used for chemotherapy, blood product 
administration, and blood drawing. Both the Broviac and Hickman Silastic catheters are appropriate not only for inpatient treatment but also for care in the outpatient setting and in the home. Thus, central lines have become a very important part of nursing management of the pediatric oncology patient (Curtas, et al., 2015).

The last decade has seen further progress in the development of new types of VADs, most notably implanted ports and peripherally inserted central catheters. Whereas this progress has revolutionized the approach to venous access in the pediatric cancer patient, we are now faced with complicated choices previously unknown to patients and care providers. Pediatric oncology nursing practice now incorporates responsibility for managing and maintaining central venous access, including establishing the best choice of VAD for the individual patient, educating patient, family, and staff in the use of VADs, and anticipating and intervening with problems that arise (Slater, et al., 2012).

This study aimed to assess the effect of nursing protocol in insertion and care of VAD in oncology pediatric patients.

The current study showed that, the majority of the studied nurses were females, and one third of them were in the age group of $25<30$ years and were diploma nurses. Regarding years of experience, almost half of them had years of experience between $5<10$ and three quarters of them didn't attend a training program regarding vascular access devices (Table $1 \&$ figure 10).

This was supported by (Boland, et al., 2013), who conducted " A randomized controlled trial to evaluate the clinical and cost-effectiveness of Hickman line insertions in pediatric cancer patients by nurses " showed that, most of the studied nurses were females, about one third of them were in the age group (25-30 years).

Also this was in agreement with the study of (Shinde, and Anjum, 2016), which was about "Educational Methods and Media For Teaching In Practice Of pediatric Nursing " clarified that, three quarters of the studied nurses didn't attain any training program regarding vascular access devices.

Regarding the Characteristics of the Studied Children the current study mentioned that, more than half of them were males, and were in primary school. Meanwhile almost one third of them were ranked as the first child in their families and their age ranged between $7-9$ years. (table $2 \&$ figure 11 ) .

This was approved by the study of (Hathorn and Pizzo, 2013), which was about "Infectious complications in the pediatric cancer patient "showed that, more than half of the studied children were males and one third of them were the first child.

Also the current work clarified that, less than half of the studied children had an illness from $3<6$ years, were diagnosed with leukemia, and were in the second stage of diseases respectively. All of them were treated with chemotherapy and performed a lumbar puncture (table 3).

This was supported by the study of (Karthaus, et al., 2016), which was about "prevention of complications in pediatric cancer patients with central venous catheters "showed that, all of the studied children were treated with chemotherapy and about half of them were diagnosed with leukemia.
Learning is the addition of new knowledge and experience. Interpreted in the light of past knowledge and experience. Teaching and learning is an integral part of nursing. Nurses have the responsibility to educate patients related to various aspects and keep themselves updated (Fridkin, et al., 2012).

The current study clarified that, there was high statistically significant difference between the studied nurses regarding their knowledge concerning types, and indications of the vascular access devices throughout the intervention (table 4 \& figure 12).

This was in agreement with (Babu, et al., 2014), who studied "Effectiveness of Planned Teaching Program on Knowledge Regarding Non-Curative Care of Terminally ILL Cancer Patients among Care Takers "showed that, there was high statistically significant difference between the studied nurses regarding their knowledge concerning types of the vascular access devices throughout the intervention.

Regarding the knowledge of the studied nurses about Port-A- cath the current work mentioned that, there was high statistically significant difference regarding their knowledge in relation to (types,meaning, indications, infection control precautions and contraindications of the Port-A-Cath) (table $5)$.

Also there was statistically significant difference regar ding their knowledge in relation to (equipment for its insertion, antiseptic solution, skin preparation, fixation methods, nursing role before, during and after its insertion, complications, steps for removal and nursing action toward the Port-A-Cath) throughout the intervention This was supported by the study of (Doenges and Murr, 2011), which was about "Nursing care plans: Guidelines for individualizing client care across the life span "mentioned that, there was high statistically significant difference $(P<0.0001)$ before and after educational Intervention guidelines regarding all items of oncology nurses Knowledge about Meaning, indication and complication of Port-a-cath.

In the study of (Maki, et al ., 2014), which was about " Prevention of central venous catheter-related bloodstream infection " showed that, there was high statistically significant difference regarding the knowledge of the studied nurses about Port-A-cath in relation to (types,indications, contraindications, nursing role for its insertion, complications, and steps of removal).

The current study mentioned that, there was statistically significant difference between the studied nurses regarding their knowledge about Hickman device concerning (types meaning, indications, instructions, infection control precautions, contraindications, antiseptic solution, nursing role before, during and after insertion, complications and reasons for removal of throughout the intervention (tables 8 - 10 \& figure 15).

This was supported by (Boland, et al., 2013), who conducted "A randomized controlled trial to evaluate the clinical and cost-effectiveness of Hickman line insertions in pediatric cancer patients by nurses " clarified that, there was statistically significant difference between the knowledge of the studied nurses about Hickman device in relation to (types, indications, contraindications, complications and infection control strategies). 
Also the current study showed that, there was statistically significant difference between the studied nurses regarding their knowledge about CVC device in relation to (meaning, indications, duration, infection control precautions,equipment, nursing role before, during and after insertion,reasons for removal and nursing action toward the complications ) throughout the intervention (tables 11- 13 \& figure 16).

This was in agreement with the study of (Krein, and Hofer, 2012), which was about "Use of central venous catheter related blood stream infection prevention practices" mentioned that, there was high statistically significant difference between the knowledge of the studied nurses about CVC device in relation to (indications, infection control precautions, complications and reasons of removal).

Staff nurses working in the clinical field are said to be backbone of the hospital. Most of the care of patients is taken by the staff nurses working in the hospital. She is the person who stays most of the time with patient and their relatives who are present in the hospital or comes in the clinic with patients. Obviously, the role of nurse is increased due to long stay with the patient. She has to take care of patients as well as she has to provide health education to patient and their relatives regarding necessary care for the prevention of complications progressive to disease (Shinde and Anjum, 2015). That's why instructed learning is so important in raising up the level of the nursing staff knowledge specially in relation to VAD.

As regard, the total knowledge of the studied nurses about vascular access devices the current study showed that, there was high statistically significant difference throughout the intervention (table 14, figure 17).

This was in the same line with the study of (Johnson, et al., 2016), which was about "Frequency of central vascular device infections in pediatric oncology patients" showed that, there was statistically significant difference between the total knowledge of the studied nurses about vascular access device.

The current study showed that, there was statistically significant difference between pre, post and follow-up intervention concerning the studied nurse's practice regarding to the preparation, insertion, and after insertion for the vascular access devices insertion (Port-A-Cath, Hickman, and CVC) throughout intervention (table $15-17$ \& figure 18-20).

Also this was supported by (Chernecky, 2011), who studied "Satisfaction versus dissatisfaction with venous access devices in pediatric oncology departments" showed that, there was statistically significant difference regarding to the studied nurse's practice concerning the preparation and the insertion of the vascular access devices insertion pre and post the intervention.

Regarding the practices of the studied nurses for Port-A-cath insertion, the current work mentioned that, there was statistically significant difference between pre, post and follow-up intervention (table 18 \& figure 21).

This was in agreement with the study of (El Malla, 2014), who studied "Improving care of children with cancer in Egypt" mentioned that, there was statistically significant difference regarding the studied nurse's practice in relation to the preparation for Port-A-Cath throughout intervention.
Vascular access devices may need to be left in place for days or even weeks; but they are associated with complications that can be mechanical or infectious. Infectious complications include bacterial or fungal sepsis. This can act as a focus for noso-comial infection that is associated with extended admission time, additional costs and increased mortality (Maki, et al., 2011).

The current work clarified that, there was statistically significant difference between pre, post and follow-up intervention concerning the studied nurse's practice regarding their daily care and maintenance of the vascular access devices (Port-A-Cath, Hickman, and CVC) throughout intervention (table 19\& figure 22).

This was in accordance with the study of (Rickard, et al., 2010), which was about "Routine re-site of peripheral intravenous devices every 3 days did not reduce complications compared with clinically indicated re-site" showed that, there was statistically significant difference regarding the practices of the studied nurses in relation to the care and maintenance of the vascular access devices pre and post intervention.

The current study mentioned that, there was statistically significant difference between pre, post and follow-up intervention concerning the studied nurse's practice regarding their flushing with heparin/ saline of the vascular access devices (Port-A-Cath, Hickman, and CVC) throughout intervention (table 20 \&figure 23).

This was supported by (Bertoglio, et al ., 2013 ), who conducted a study about "Pre-filled normal saline syringes to reduce totally implantable venous access device-associated bloodstream infection " showed that, there was high statistically significant difference pre \& post intervention, regarding the practices of the studied nurses in relation to flushing with saline of the vascular access devices.

Also this was approved by the study of (Fernandez, et al., 2012), which was about "Peripheral venous catheters: a review of current practices " clarified that, there was statistically significant difference regarding the nurses practices concerning the flushing of VAD with heparin / saline.

Regarding the practices of the nurses in relation to obtaining blood specimens from vascular access devices, the current study showed that, there was statistically significant difference between pre, post and follow-up intervention (table $21 \&$ figure 24). This was in agreement with (Frey, 2011), who studied "Drawing Blood Samples from Vascular Access Devices: Evidence-Based Practice," Journal of Infusion Nursing " showed that, there was statistically significant difference pre, and post intervention regarding the nurses practices in relation to obtaining blood specimens from vascular access devices.

Also the current work revealed that, there was statistically significant difference between pre, post and followup intervention concerning studied nurse's practice regarding their administration of fluid infusion and chemotherapy through the vascular access devices (Port-A-Cath, Hickman, and CVC) throughout intervention (tables 22,23 \& figures 25,26).

In the study of (Vescia, et al., 2011), who studied "Management of venous port systems in oncology" showed that, regarding the practices of the studied nurses in relation to administration of chemotherapy through 
vascular access devices, there was statistically significant difference between pre, post intervention.

The current study showed that, there was statistically significant difference between pre, post and follow-up intervention in relation to the studied nurse's practice regarding to antibiotic lock for the vascular access devices (Port-A-Cath, Hickman and CVC) and their removal throughout intervention (tables 24,25 \& figures $27,28)$.

This was in agreement with (Bagnall-Reeb and Ruccione, 2010 ), who conducted a study about " Management of cutaneous reactions and mechanical complications of central venous access devices in pediatric patients with cancer " mentioned that, there was statistically significant difference between pre, and post intervention regarding the nurses practices in relation to removal of VAD.

As regard the total practices of the studied nurses in relation to vascular access devices (Port-A-Cath, Hickman, and CVC), the current work reported that, there was statistically significant difference throughout intervention (table 26 \& figure 29).

This was in accordance with (Leung, et al., 2011), who conducted "A retrospective study on the long-term placement of peripherally inserted central catheters and the importance of nursing care and education " mentioned that,, it was observed that, there was marked improvement in nurses' total knowledge after implementation of the educational Intervention guidelines with high statistically significant difference.

Also this was supported by the study of (Dearborn, et al., 2013); who studied "Nurse and patient satisfaction with three types of venous access devices" reported that, the total knowledge of the studied nurses improvement in a satisfied way after implementation of educational programs with statically significant difference.

The current work showed that, there was positive correlation between knowledge of the studied nurses and their total practices, regarding vascular access devices (Port-A-Cath, Hickman, and CVC) throughout intervention with statically significant difference (table 27).

This was supported by (lkubati, et al., 2015), whose study was about "Health care workers' knowledge and practices regarding the prevention of central venous catheter-related infection" mentioned that, there was a positive correlation between the knowledge of the health care workers specially the nursing staff and their practices, regarding the vascular access devices.

\section{CONCLUSION}

Based on finding of present study, it can be concluded that: Implementation of the nursing protocol patients has statistically significant positive effect in insertion and care of VAD on oncology pediatric patients

\section{Recommendation:}

Based on the finding of this study, the following recommendation was inferred from the study:

Recommendation related to adolescents:

- Replication of the current study on larger probability sample is recommended to achieve generalization of the results and winder utilization of the nursing protocol

- $\quad$ the nursing protocol should be applied for all nurses who deals with pediatric devices

- $\quad$ Further research are recommended related to improve nurses performance related pediatric devices

\section{REFERENCES}

1. Babu, R. L., Mali, N., and Shinde, M. Effectiveness of Planned Teaching Program on Knowledge Regarding Non-Curative Care of Terminally ILL Cancer Patients among Care Takers. International Journal of Science and Research (IJSR), 3(4), 2014, 198-205

2. Bagnall-Reeb HA, and Ruccione K: Management of cutaneous reactions and mechanical complications of central venous access devices in pediatric patients with cancer: Algorithm for decision making. Oncol Nurs Forum 17 : 677- 681, 2010

3. Barnes, J.; Pressey, J.; Adams, J.; Hensler, A. and MadanSwain, A. Weiser. Physician and nurse beliefs of phase 1 trials in pediatric oncology. Cancer Nursing, 2014, 37(5): E48-E52.

4. Bertoglio, S., Rezzo, R., and Merlo, F.D. Pre-filled normal saline syringes to reduce totally implantable venous access deviceassociated bloodstream infection: a single institution pilot study. J. Hosp. Infect. 2013, 84 (1), 85-88

5. Boland A, Haycox A, Bagust A, and Fitzsimmons L. A randomised controlled trial to evaluate the clinical and costeffectiveness of Hickman line insertions in pediatric cancer patients by nurses. Health Technol Assess 2013; 7: 1-99

6. Braveman, D.; Gil, K.; King, C. and Piatt, E. Chon. Clinical outcomes in a primary care practice within a center for health equity. Journal of Primary Care and Community Health, 2012, 2150131915583658.

7. Chernecky C. Satisfaction versus dissatisfaction with venous access devices in pediatric oncology departments: a pilot study.; Oncology Nursing Forum. 2011; 28(10):1613-6

8. Curtas $S$, Bonaventura $M$, and Mëguid $M$ : Cannulation of inferior vena cava for central venous access. Surg Gynecol Obstet 168:121-124, 2015

9. Dearborn P, De Muth JS, Requarth $A B$, and Ward SE; Nurse and patient satisfaction with three types of venous access devices; Oncology Nursing Forum. 2013; 24 (1 Suppl): 34-40.

10. Doenges, M. and Murr, A. Nursing care plans: Guidelines for individualizing client care across the life span, (8th ed.), Published by F.A Davis Company, 2011.

11. El Malla, H. Improving care of children with cancer in Egypt. National Cancer Institute (Egypt), 2014.

12. Fernandez, R.S., Griffiths, R.D., and Murie, P. Peripheral venous catheters: a review of current practices. J. Infus. Nurs. 2012, 26 (6), 388-392

13. Frey, A. "Drawing Blood Samples from Vascular Access Devices: Evidence-Based Practice," Journal of Infusion Nursing. 26(5):285-293, September-October 2011

14. Fridkin S.K., Pear S.M., Williamson T.H., Galgiani J.N., and Jarvis W.R. The role of understaffing in central venous catheterassociated bloodstream infection. Infection Control and Hospital Epidemiology. 2012, 17(3): 150-158. March.

15. Gallieni M., Pittiruti M., Biffi R. Vascular access in oncology patients. CA Cancer J Clin 2018; 58: 323-346.

16. Hathorn JW, and Pizzo PA. Infectious complications in the pediatric cancer patient. Philadelphia, PA, Lippincott, 2013, pp 837-86.

17. Hickman R, Buckner C, and Clift R. A modified right atrial catheter for access to the venous system in marrow transplant recipients. Surg Gynecol Obstet 148:871-875, 2013.

18. Johnson E, Babb $J$ and Sridhar D. Routine Antibiotic Prophylaxis For Totally Implantable Venous Access Device Placement: Meta-Analysis Of 2,154 Patients. J Vasc Interv Radiol 2016; 27(3): 339-343.

19. Karthaus $M$, Kretzschmar $A$, and Kröning $H$. prevention of complications in pediatric cancer patients with central venous catheters: final results of a double-blind, placebo controlled phase III trial. Ann Oncol 2016; 17: 289-296 
20. Krein, and Hofer, Use of central venous catheter related blood stream infection prevention practices by U.S.hospitals, 2012, 82(6), 672-678

21. Leung, T. K., Lee, C. M., Tai, C. J., Liang, Y. L., and Lin, C. C. (2011): A retrospective study on the long-term placement of peripherally inserted central catheters and the importance of nursing care and education. Cancer Nursing, 34 (1).

22. Ikubati SA, Ahmed NT, Mohamed on, Fayed AM, and Asfour HI. Health care workers' knowledge and practices regarding the prevention of central venous catheter-related infection. Am J Infect Control 2015;43:26-30.

23. Maki D.G., Stoltz S.M., Wheeler S., and Mermel L.A. Prevention of central venous cathter-related bloodstream infection by use of an antiseptic-impregnated catheter: a randomised, controlled trial. Annals of Internal Medicine. 2014, 127(4): 257-266.

24. Maki, D.G., Kluger, D.M., Crnich, and C.J. The risk of blood stream with different intravascular devices: a systematic review of 200 published prospective studies. Mayo Clin. Proc.2011, 81 (9), 1159-1171.

25. Randolph, A. G., Cook, D. J., Gonzales, C. A., \& Andrew, M.
Benefit of heparin in peripheral venous and arterial catheters: Systematic review and meta-analysis of randomized controlled trials. BMJ (Clinical Research ed.), 2013, 316 (7136), 969-975. doi: 10.1136/ bmj. 316. 7136.969

26. Rickard, C.M., McCann, D., Munnings, J., and McGrail, M.R., Routine resite of peripheral intravenous devices every 3 days did not reduce complications compared with clinically indicated resite: a randomised controlled trial. 2010, BMC Med. 8, 53

27. Shinde, M., and Anjum, S. Educational Methods and Media For Teaching In Practice Of pediatric Nursing. Sneha Publication India (Dombivili), 2016.

28. Slater H, Goldfarb I, and Jacob H: Experience with long-term outpatient venous access utilizing percutaneously placed silicone elastomer catheters. Cancer 56:2074-2077, 2012

29. Strootman, V. Intravenous and vascular access therapy. In A. G. Perry, P. A. Potter, \& W. R. Ostendorf (Eds.), Clinical nursing skills \& techniques (8th ed., pp. 724-735). St. Louis, MO: Mosby Elsevier, 2014.

30. Vescia S, Baumgärtner AK, and Jacobs VR. Management of venous port systems in oncology: a review of current evidence. Ann Oncol 2011;19:9-15. 